\title{
Nanomedicine lacks recognition in Europe
}

A recent report by the European Science Foundation (ESF) considers what action is needed to foster medical applications of nanotech in Europe. Nanomedicines, the report claims, are still facing hurdles related to lack of an adequate funding structure and to potential difficulties in gaining regulatory approval. Those with experience in developing this type of treatment argue, however, that any safe and effective nanomedicine would make it to the market without the need for additional regulations.

The report 'Forward-Look Study on Nanomedicine' published in December 2005 presents the conclusions of an expert group set up by the ESF to provide government agencies and policy makers with a map that will enable them to take a strategic approach to investing in nanomedicine. This field has now reached a significant size. According to figures compiled by the US industry newsletter NanoBiotech News, more than 130 nanotech-based drugs and delivery systems and 125 devices or diagnostic tests are in preclinical, clinical or commercial development. Those figures are up from the 61 drugs and 91 devices a year earlier.

The first task for the 30 industrialists and academics who compiled the report was to define the field. Their vision is of a distinct sector, drawing on molecular tools and molecular knowledge to translate the increase in understanding of the cellular basis of pathophysiology into overall improvements in healthcare. But the ESF report points out that for nanotech to reach its full potential, disparate elements, ranging from proteomics, genomics, drug delivery and diagnostics and imaging, need to be pulled together. "One of the key challenges will be bringing together the interdisciplinary components and getting them to work together in true collaborations," comments Ruth Duncan, who chaired the investigation, and who is the director of the center for polymer therapeutics at Cardiff University, in Wales.

The proponents of nanomedicine claim that the power of nanotech lies in its ability to operate on the same atom-by-atom scale as all the intimate biochemical functions that are involved in the growth, development and aging of the human body. However, this could be a double-edged sword, presenting problems in getting regulatory approvals. Wolfgang Kreyling, group leader at the Institute for Inhalation Biology in Munich, points out that the nature of nanoparticles means there may be unexpected physical or chemical interac-

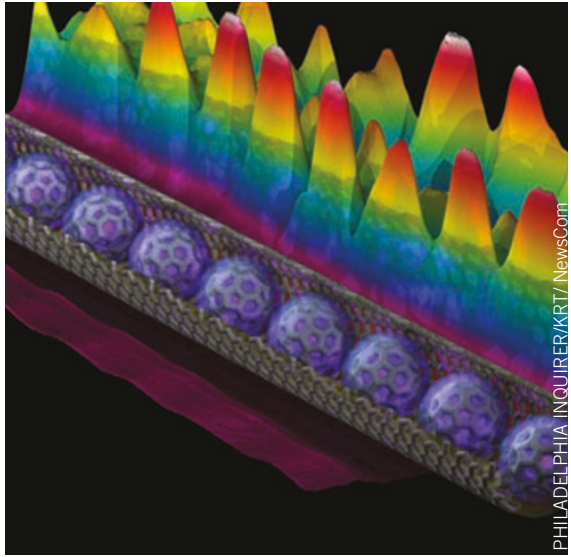

Beside creating scientific methodologies for assessing such technologies, there is a need for funding institutions to start allocating resources to an area that has, so far, not been recognized in its own right. Indeed, despite the ESF's report, and an earlier one, 'European Technology Platform on Nanomedicine' published by the European Union's research directorate in September 2005, nanomedicine will not be funded as a coherent discipline in the upcoming Framework 7 program.

Responding to the report, Renzo Tomellini, head of the nanotech unit at the European Commission in Brussels confirms that 'nanotech' and 'healthcare' will be separate themes in the Framework 7 program, due to start in January 2007. By comparison, in the US the field has recently received funding through the five-year, \$144-million nanomedicine plan of the National Cancer Institute and the \$42-million plan of the National Institutes of Health to set up four nanomedicine development centers.

These observations regarding issues of funding and regulatory approval are somewhat at odds with the fact that Appendix $\mathrm{V}$ of the report lists a number of registered nanomedicines (Box 1). Mike Eaton, head of antibody chemistry at Brussels-based biopharmaceutical company UCB Celltech comments, "I'm not sure you need new regulation. Nanomedicines are not new; they have been getting regulatory approval for ten years."

He adds, "The 'nano' element has not been a feature; each drug is taken on its merits." In the US, the FDA has taken a similar view, and assessed nanotech-based candidate drugs on a case-by-case basis. Eaton, however, admits he has concerns about what he termed "exotic" constructs using carbon nanoparticles. "But if there are problems they won't get there [approved]. If they are genuine advances, and they are safe, they will."

Nuala Moran, Cheshire, UK

\section{Box 1 Nanomedicines already approved}

There are already biotech applications of nanomedicines on the market. These include polymer-protein conjugates that are polyethylene glycol (PEG)-modified (pegylated) versions of existing drugs, such as PEG-granulocyte colony stimulating factor (Amgen's Neulasta). What's more, "Diagnostics are starting to come through, and real-time imaging will come through soon," says another contributor to the report, Julie Deacon, consultant at the government-funded group UK Micro and Nanotechnology Network. Imaging agents that highlight biochemical processes in real time may also follow marketed products, such as the Guerbet Group's Endorem, a magnetic resonance imaging contrast agent consisting of superparamagnetic iron oxide nanoparticles. 